\title{
Emergency Materials Supply Chain Coordination by the Joint Mechanism between Government and Enterprises
}

\author{
Bin Ding $^{1} \&$ Yufeng Huang ${ }^{1}$ \\ ${ }^{1}$ School of Management, University of Science and Technology of China, Hefei, China \\ Correspondence: Yufeng Huang, School of Management, University of Science and Technology of China, No. \\ 919, Manage School building, East Campus, USTC, Hefei, China. E-mail: huangyuf@mail.ustc.edu.cn
}

\author{
Received: March 14, $2012 \quad$ Accepted: April 17, $2012 \quad$ Published: July 1, 2012 \\ doi:10.5539/ass.v8n8p15 \\ URL: http://dx.doi.org/10.5539/ass.v8n8p15
}

Foundation: National Natural Science Foundation of China(71171182)

\begin{abstract}
This paper researches into the sales of co-reserve emergency supplies by government and enterprises united, in which the government ( by commission, holding a company) ordered emergency supplies from the Suppliers and updates the selling dynamically within the validity period of the quality of the materials. It also analyzes that the demand change caused by the change of the sensitivity coefficient of the price-demand function under unexpected emergencies may lead to the failure of the coordination of the original supplier-government-retailer three-tier supply chain and the paper also gives the optimal strategy for the supply chain to respond to the emergencies, and puts forward the corresponding quality discounts and revenue sharing contract. Finally, the equation used in this paper shows that this mode of cooperation can not only reduce the government budget, but also has the anti-emergencies ability.
\end{abstract}

Keywords: emergency materials, coordination mechanism, government and enterprise joint reserve sales, cooperative game, revenue sharing contract

\section{Introduction}

The 9.21 Taiwan earthquake in the year 1999, the 9.11 terrorist event in 2001, the SARS crisis in 2003, the 2011 Japanese earthquake and tsunami and etc, these emergencies or natural disasters not only bring damage to the society, but also have a big effect on the market demand of the supply chain, production arrangement, distribution and logistics and other aspects. After the emergencies, the original plan of the supply chain members may be disturbed, the contract concluded between the stakeholders may no longer apply to the whole supply chain coordination, and the national macro-control is not only laborious, but also can't fundamentally solve the problem. In order to reduce the casualties and property losses caused by the disasters as much as possible, it is particularly important for us to do the procurement of the emergency supplies and meanwhile coordinate the anti-emergencies of the contract.

In the traditional non-contractual supply chain, enterprises in the supply chain only focus on how to maximize their profits, while ignoring the overall profit of the supply chain (Chen et al., 2007, p.585-590; Yang et al., 2007, p.1-5; Wang et al., 2006, p.522-525; Xin et al., 2007, p.1687-1692). Pasternack first proposed the concept of supply chain contracts. Since then, scholars carry out a lot of researches on the supply chain contract which have already made considerable development in some aspects (Pasternack, 1985, p.166-176). Clark et al., extended it to a multi-stage inventory, and through dynamic programming method established a relatively independent single product optimal order quantity and trading strategies with the same demand distribution, and at the same time extended it to the downstream retailers, which can be seen as the prototype of the supply chain contract (Clark \& Scarf, 1960, p.475- 490). Zhang Handong et al., based on the ideas of inventory loss and inventory related to time, considered the economic order quantity of the quality discount perishable goods under the Elastic demand conditions (Zhang etc., 2009, p.1528-1533). Lei Dong et al., analyzed a linear function of the demand 
for the price, the scale of demand and production costs changes at the same time, and how to use quantity discounts contract to conduct Stackelberg game coordination (Lei et al., 2006, p.51-59). Yang Zhihui etc., analyzed when the retailers faced with stochastic demand distribution function, how could the quantity discount contract respond to the emergerncies of the simultaneous changing of the demand and cost (Yang et al., 2010, p.728-732). Cachon pointed out that: a supply chain called a treaty mechanism is coordinated through the treaty, if through this treaty, the optimal solution of the supply chain is the Nash equilibrium point which composes the business decisions in the supply chain, namely: If in the process, when the supplier and retail business pursue their own revenue maximization, meanwhile the supply chain gets the maximal return, both sides can get more benefits without the loss of each other's interests (Cachon, 2010, p.229-339). Jeuland pointed out that the supply chain is coordinated under the quantity discount contract, if: For any $\eta: 0<\eta<1$, can get $\bar{f}^{r}=(1-\eta) \bar{f}^{s c}$, that is the retailer's revenue is the revenue function of the supply chain linear (affine) function (Jeuland \& Shugan, 1983, p.239-272). De-Li Yang et al., summarized, analyzed, and inducted the origin and development of supply chain contract, and then classified the current researches of the supply chain contract, and established the basic mathematical model for several typical supply chain contract. On this basis, they further explored the mechanism of action and the coordination methods of supply chain contract in the supply chain coordination, and finally concluded the development trend of the supply chain contract (Yang et al., 2006, p.117-125).

According to the general law of the market, it is assumed that market demand $d$ is a decreasing function of the sales price (Qi et al., 2004, p.301-312): $d=D-k p$, in the function, $D$ represents the scale of market demand (ie: the maximum demand of the market), $k>0$ is the sensitivity coefficient of the demand for the price function, $d$ is the practical needs under the retail price $p$.

The above researches are for the two-stage supply chain, while it is more common for us to see the multi-stage supply chain in the real process. This article is to break the single concentrated reserve mode of the traditional emergency supplies, and to reserve and sell the emergency supplies by the government and enterprises united, that is the government (through the commission, holding a company) orders emergency supplies from the suppliers and update the selling dynamically within the validity period of the quality of the goods., thereby establishing the three-tier supplier - Government - retailers supply chain. This paper researches into the optimal decision responding to emergencies taking into account the changing of the sensitivity coefficient of the price demand function lead by the unexpected events, and put forward the corresponding discounts and benefits sharing contract. Finally, through example, this paper shows that the coordination mechanism can not only reduce the government budget, but also has the anti-emergencies ability.

\section{The Basic Model}

In this paper, we research the three-tier supply chain by the supplier - government - retailer based on volume discounts and revenue sharing. As shown:

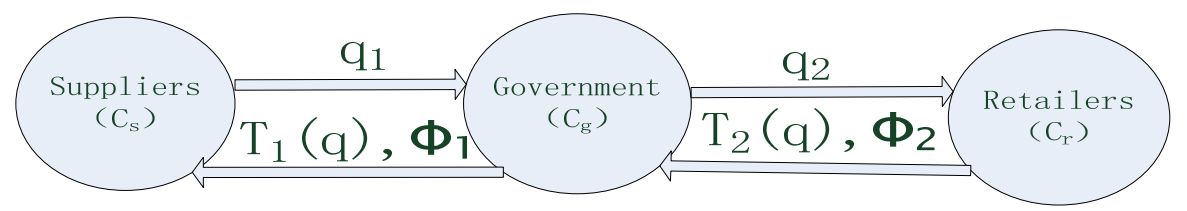

Figure 1. The number of discounts and revenue sharing of coordination mechanism in three supply chain

$q_{1}$ : According to market forecasts, the government submitted the number of ordered goods to supplier;

$q_{2}$ : According to market forecasts, retailer submitted the number of ordered goods to the government;

$d:$ the demand of retailer;

$C_{s}$ : Unit marginal cost of supplier (Including the cost of production and transportation costs);

$C_{g}$ : Unit marginal cost of government (Including management, inventory and transportation costs);

$C_{r}$ : Unit marginal cost of the retailer (Including sales and transportation costs); 
$C=C_{s}+C_{g}+C_{r}$

$\Phi_{1}$ : Government returned $1-\Phi_{1}\left(0 \leq \Phi_{1} \leq 1\right)$ of its income to the supplier;

$\Phi_{2}$ : Retailer returned $1-\Phi_{2}\left(0 \leq \Phi_{2} \leq 1\right)$ of its income to the government;

$T_{1}(q)$ : The payment which the Government gives to the supplier;

$T_{2}(q)$ : The payment which the Retailers gives to the government;

$p:$ The market price per unit of goods;

Above all, the profits of supplier are affected by the retailer's pricing decisions, although the supplier does not directly deal with the retailer, the supplier can influence retailer indirectly by the government. The relationship of the adjacent members can be seen as Stackelberg game. We assume that the information is perfectly symmetrical, the supplier, government and retailer are risk-neutral and perfectly rational, they make decisions independently in order to make their profit maximal. The number of the products that the government or retailer ordered are consistent with the market demand, denoted as: $q_{1}=q_{2}=d=q$.

While ordered $q$, the payment which the government gives to the supplier is $T_{1}(q)$, The payment which the retailer gives to the government is $T_{2}(q)$, the supplier's profit is: $\overline{\prod_{s}(q)}=\mathrm{T}_{1}(q)-C_{s} q$, the government's profit is: $\overline{\prod_{g}(q)}=\mathrm{T}_{2}(q)-\mathrm{T}_{1}(q)-C_{g} q$, the retailer's profit is: $\overline{\prod_{r}(q)}=p q-\mathrm{T}_{2}(q)-C_{r} q$, the supply chain profit is: $\overline{\prod_{(q)}}=\overline{\prod_{s}(q)}+\overline{\prod_{g}(q)}+\overline{\prod_{r}(q)}$, owing to $q_{1}=q_{2}=d=q$ and $d=D-k p, p=(D-q) / k$. Then $\overline{\prod(q)}=(p-C) q=q[(D-q) / k-C]$, using simply algebra, we know that when $\bar{p}=(D+\mathrm{k} C) / 2 k$, the supply chain profit is maximized. Substituting this value into the former expression gives $\overline{\prod_{\max }(q)}=(D-k C)^{2} / 4 k ;$ the corresponding production is $\bar{q}=(D-k C) / 2 \cdot$

There are two problems in implementing a coordination scheme that will achieve the maximum supply chain profit:(1) how to share the profit among the supplier, government and retailer and (2) how to compel the retailer to order $\bar{Q}$ and set the retail price at $\bar{p}$.

In the case of revenue sharing, government returned $1-\Phi_{1}\left(0 \leq \Phi_{1} \leq 1\right)$ of its income to the supplier; retailer returned $1-\Phi_{2}\left(0 \leq \Phi_{2} \leq 1\right)$ of their income to the government; The entire supply chain process is shown in Figure 1. Based on the above description we can see that the expected revenue functions of the retailer, the government and the supplier are respectively as follow:

$$
\left\{\begin{array}{l}
\overline{\prod_{r}(q)}=\Phi_{2} p q-\mathrm{T}_{2}(q)-C_{r} q \\
\overline{\prod_{g}(q)}=\Phi_{1}\left[\mathrm{~T}_{2}(q)+\left(1-\Phi_{2}\right) p q\right]-\mathrm{T}_{1}(q)-C_{g} q \\
\overline{\prod_{s}(q)}=\left(1-\Phi_{1}\right)\left[\mathrm{T}_{2}(q)+\left(1-\Phi_{2}\right) p q\right]+\mathrm{T}_{1}(q)-C_{s} q \\
\overline{\prod_{(}(q)}=p q-C q=(p-C) q
\end{array}\right.
$$

In order to coordinate the above supply chain system, We use a quantity discount contract to find $W_{1}(q)$ and $W_{2}(q)$ appropriately, make $T_{1}(q)=W_{1}(q) \cdot q$ and $T_{2}(q)=W_{2}(q) \cdot q$. Under normal circumstances, $W_{1}(q)>C_{s} ; W_{2}(q)>W_{1}(q)+C_{g}$; retail price $p>W_{2}(q)+C_{r}$. 
Theorem 1. For any $\Phi_{1}, \Phi_{2} \in[0,1]$, suppose $W_{1}(q)=\Phi_{1}\left(C_{s}+C_{g}\right)-C_{g}$ and $W_{2}(q)=\Phi_{2} C-C_{r}$, then

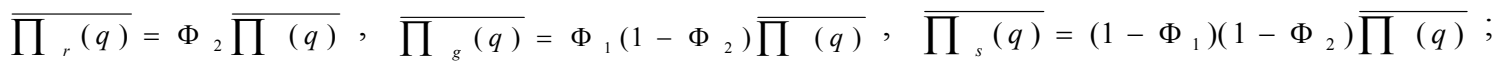

which can be expressed as, the supply chain has been coordinated at the basis of benefit-sharing contract and quantity discount contract.

Simultaneously, we should allocate $\Phi_{1}$ and $\Phi_{2}$ rationally to make $W_{1}(q)=\Phi_{1}\left(C_{s}+C_{g}\right)-C_{g} \geq 0$, $W_{2}(q)>0$, So there: $\Phi_{1}>C_{g} /\left(C_{s}+C_{g}\right), \Phi_{2}>C_{r} / C$.

Proof of Theorem 1. For any $\Phi_{1}, \Phi_{2} \in[0,1], W_{1}(q)=\Phi_{1}\left(C_{s}+C_{g}\right)-C_{g}$ and $W_{2}(q)=\Phi_{2} C-C_{r}$ :

$$
\left\{\begin{array}{l}
\overline{\prod_{r}(q)}=\Phi_{2} p q-\mathrm{T}_{2}(q)-C_{r} q=\Phi_{2}(p-C) q=\Phi_{2} \overline{\prod(q)} \\
\overline{\prod_{g}(q)}=\Phi_{1}\left[\mathrm{~T}_{2}(q)+\left(1-\Phi_{2}\right) p q\right]-\mathrm{T}_{1}(q)-C_{g} q=\Phi_{1}\left(1-\Phi_{2}\right) \overline{\prod(q)} \\
\overline{\prod_{s}(q)}=\left(1-\Phi_{1}\right)\left[\mathrm{T}_{2}(q)+\left(1-\Phi_{2}\right) p q\right]+\mathrm{T}_{1}(q)-C_{s} q=\left(1-\Phi_{1}\right)\left(1-\Phi_{2}\right) \overline{\prod(q)}
\end{array}\right.
$$

In other words, with the quantity discounts and revenue sharing contract, the revenue of retailer and government becomes a radiation function of the payoff function of the entire supply chain. In that case, the retailer and Government's optimal order quantity is also a system optimal order quantity, and we can adjust $\Phi_{1}, \Phi_{2}$ to allocate the revenue of the entire supply, therefore, the supply chain is coordinated.

\section{Supply Chain Coordination Mechanism to the Centralized Decision-making with Emergencies}

\subsection{The Centralized Supply Chain Coordination Model}

From the above, The supply chain can maximize the benefits. Here we consider unexpected events happening after the supplier's production schedule arranged. Because the demand change caused by the change of the sensitivity coefficient of the price-demand function under unexpected emergencies may lead to the failure of the coordination of the original three-level supply chain composed by the supplier-government-Retailer. Suppose the sensitivity coefficient of the price demand function that retailer faced change into $k+\Delta k$, retail price is $p$, the demand that retailer faced change into $d=D-(k+\Delta k) p$.

After the event of emergencies, we see government as a centralized decision-maker who seeks to maximize the total supply chain profit. Let $Q$ be the real demand under the resolved demand-price relationship, and $p$ the new retail price, where $p=(D-Q) /(k+\Delta k)$. Now the real demand $Q$ is equal to the original production plan as $\bar{Q}=\bar{q}$. Let $\Delta Q=Q-\bar{Q}$, when $\Delta Q<0$, Production is more than demand, and the excess production have to be scrapped or possibly sold in a secondary market at a price much below $p$; when $\Delta Q>0$, Production must be increased to satisfy the new demand. Quite often, due to the need to use more expensive resources, the unit production cost associated with the additional demand will be more than the unit production cost associated with the base demand.

From the central decision-maker's point of view, given the production quantity $Q$, the supply chain profit under emergencies can be written as

$$
\prod(Q)=Q\left(\frac{D-Q}{k+\Delta k}-C\right)-\lambda_{1}(Q-\bar{Q})^{+}-\lambda_{2}(\bar{Q}-Q)^{+}-\lambda(Q-\bar{Q})^{2}
$$

Where the parameters $\lambda_{1}, \lambda_{2}, \lambda>0 \quad$ and $A^{+}=\max \{0, A\}$. more precisely, $-\lambda_{1}(Q-\bar{Q})^{+}-\lambda_{2}(\bar{Q}-Q)^{+}-\lambda(Q-\bar{Q})^{2}$ is additional cost which caused by breaking the original 
production plan, $\lambda_{1}>0$ is the cost incurred by the supplier in addition to $C$ when $\Delta Q=Q-\bar{Q}>0$, and $\lambda_{2}>0$ is the disposal cost per unit when $\Delta Q=Q-\bar{Q}<0$. When $0<\lambda_{2}<C$, the model represents the case in which the surplus $\bar{Q}-Q$ can be sold in a secondary market at a price less than $C$, say, $\bar{C}=C-\lambda_{2}$. For $\lambda(Q-\bar{Q})^{2}$, it is another cost which caused by breaking the original production plan, and is a Quadratic nonlinear function about $\Delta Q$. such as: Supplier need to sign a labor contract with workers after the original production plan, whether to increase production or sell the excess products in a secondary market, the supplier need to break the labor contract, and increase the cost.

It is clear that, when $k+\Delta k \leq 0$ the retailer can sell products at the number of $D$ by any price, That is impossible in reality, So consider $k+\Delta k \geq 0$, and we have the following theorem,

Theorem 2. Suppose $Q^{*}$ maximizes $\prod(Q)$ in (1), Then $Q^{*} \geq \bar{Q}$ if $-k<\Delta k<0$, and $Q^{*} \leq \bar{Q}$ if $\Delta k>0$.

Proof of Theorem 2. We just need to proof $Q^{*} \geq \bar{Q}$ if $-k<\Delta k<0$. The second conclusion can be similar to be proved.

Use of apagoge, Suppose $Q^{*}<\bar{Q}$ when $-k<\Delta k<0$.

As for the original optimal order quantity $\bar{Q}$,

$$
\Pi(\bar{Q})=\bar{Q}\left(\frac{D-\bar{Q}}{k+\Delta k}-C\right)=\bar{Q}\left(\frac{D-\bar{Q}}{k}-C\right)-\frac{\Delta k}{k(k+\Delta k)}(D-\bar{Q}) \bar{Q}
$$

And

$$
\overline{\Pi\left(Q^{*}\right)}=Q \cdot\left(\frac{D-Q}{k}-C\right) \leq \bar{Q}\left(\frac{D-\bar{Q}}{k}-C\right)=\overline{\prod(\bar{Q})}=\overline{\prod_{\max }(q)}
$$

Let $G(Q)=(D-Q) Q$. Obviously, the maximum value is $\hat{Q}=D / 2$, and $G(Q)=(D-Q) Q$ is an increasing function at $[0, D / 2]$, what's more, $Q^{*}<\bar{Q}<D / 2$.

So $G\left(Q^{*}\right)<G(\bar{Q})$, which can be expressed as:

$$
\left(D-Q^{*}\right) Q^{*}<(D-\bar{Q}) \bar{Q}
$$

And because $-k<\Delta k<0$, we have

$$
-\Delta k / k(k+\Delta k)>0
$$

From (4) and (5) we know: $-\Delta k\left(D-Q^{*}\right) Q^{*} / k(k+\Delta k)<-\Delta k(D-\bar{Q}) \bar{Q} / k(k+\Delta k)$, and then:

$$
\begin{aligned}
\prod\left(Q^{*}\right) & =Q^{*}\left(\frac{D-Q^{*}}{k+\Delta k}-C\right)-\lambda_{2}\left(\bar{Q}-Q^{*}\right)-\lambda\left(Q^{*}-\bar{Q}\right)^{2} \\
& =Q^{*}\left(\frac{D-Q^{*}}{k}-C\right)-\frac{\Delta k}{k(k+\Delta k)}\left(D-Q^{*}\right) Q^{*}-\lambda_{2}\left(\bar{Q}-Q^{*}\right)-\lambda\left(Q^{*}-\bar{Q}\right)^{2} \\
& \leq \bar{Q}\left(\frac{D-\bar{Q}}{k}-C\right)-\frac{\Delta k}{k(k+\Delta k)}(D-\bar{Q}) \bar{Q}-\lambda_{2}\left(\bar{Q}-Q^{*}\right)-\lambda\left(Q^{*}-\bar{Q}\right)^{2} \leq \prod(\bar{Q})
\end{aligned}
$$

It contradict with that $Q^{*}$ maximizes $\prod(Q)$ in (1), So the conclusion is proved.

From theorem 2, when $-k<\Delta k<0$, the problem of maximizing $\prod(Q)$ reduces to maximizing the 
strictly concave function.

$$
\prod_{1}(Q)=Q\left(\frac{D-Q}{k+\Delta k}-C\right)-\lambda_{1}(Q-\bar{Q})-\lambda(Q-\bar{Q})^{2}
$$

Subject to $Q \geq \bar{Q}$. Using the first-order necessary (and sufficient)condition $\prod_{1}(Q)^{\prime}=0$ for an unconstrained problem, Solving for $Q$ gives

$$
Q_{1}=\bar{Q}-\frac{\left(C+\lambda_{1}\right) \Delta k+\lambda_{1} k}{2 \lambda(k+\Delta k)+2}
$$

For constraint $Q \geq \bar{Q}$, need $\frac{\left(C+\lambda_{1}\right) \Delta k+\lambda_{1} k}{2 \lambda(k+\Delta k)+2} \leq 0$, which can be expressed as $\Delta k \leq-\frac{\lambda_{1} k}{C+\lambda_{1}}$.

When $-k<\Delta k \leq-\frac{\lambda_{1} k}{C+\lambda_{1}}, Q_{1}$ meet $Q \geq \bar{Q}$, shown in Figure 2, so $Q_{1}$ maximizes $\prod_{1}(Q)$. Let $\eta_{1}=\frac{\left(C+\lambda_{1}\right) \Delta k+\lambda_{1} k}{2 \lambda(k+\Delta k)+2}$ and $Q_{1}^{*}=Q_{1}=\bar{Q}-\eta_{1}$, corresponds to optimal retail price of the optimal order quantity $Q_{1}^{*}$ is: $p_{1}^{*}=\frac{D-Q_{1}^{*}}{k+\Delta k}=\bar{p}+\frac{\eta_{1}-\Delta k \bar{p}}{k+\Delta k}$ : optimal profit is:

$$
\begin{aligned}
\prod\left(Q_{1}^{*}\right) & =Q_{1}^{*}\left(p_{1}^{*}-C\right)+\lambda_{1} \eta_{1}-\lambda \eta_{1}{ }^{2} \\
& =\overline{\prod_{\text {max }}(q)}+\left(\bar{Q}-\eta_{1}\right) \frac{\eta_{1}-\Delta k \bar{P}}{k+\Delta k}-\eta_{1}(\bar{P}-C)+\lambda_{1} \eta_{1}-\lambda \eta_{1}{ }^{2}
\end{aligned}
$$

When $-\frac{\lambda_{1} k}{C+\lambda_{1}}<\Delta k \leq 0, Q_{1}<\bar{Q}$, shown in Figure 3, $Q_{1}$ is not feasible. According to Figure 3: $\prod_{1}(Q)$ is monotonically decreasing at $[\bar{Q},+\infty], \bar{Q}=\frac{D-k C}{2}$ maximizes $\prod_{1}(Q)$. Let $Q_{2}^{*}=\bar{Q}$, the corresponding optimal retail price is $p_{2}^{*}=\frac{D-Q_{2}^{*}}{k+\Delta k}=\frac{D-\bar{Q}}{k+\Delta k}=\bar{p}-\frac{\Delta k \bar{p}}{k+\Delta k}$, the optimal profit of the supply chain is

$$
\prod\left(Q_{2}^{*}\right)=Q_{2}^{*}\left(p_{2}^{*}-C\right)=\bar{Q}\left(\bar{P}-\frac{\Delta k \bar{P}}{k+\Delta k}-C\right)=\overline{\prod_{\max }(q)}-\frac{\Delta k \bar{P}}{k+\Delta k} \bar{Q}
$$

As is similar to $\Delta k<0$, when $\Delta k>0$, the problem of maximizing $\prod(Q)$ reduces to seek Optimal solution of the following problem:

$$
\prod_{2}(Q)=Q\left(\frac{D-Q}{k+\Delta k}-C\right)-\lambda_{2}(\bar{Q}-Q)-\lambda(Q-\bar{Q})^{2}
$$

Subject to $\bar{Q} \geq Q$, Using the first-order necessary (and sufficient)condition $\prod_{2}(Q)^{\prime}=0$ for an unconstrained problem, Solving for $Q$ gives: 


$$
Q_{2}=\bar{Q}-\eta_{2}\left(L \text { et } \quad \eta_{2}=\frac{\left(C-\lambda_{2}\right) \Delta k-\lambda_{2} k}{2 \lambda(k+\Delta k)+2}\right)
$$

For constraint $\bar{Q} \geq Q$, need $\eta_{2}>0$, Which can be expressed as $\Delta k \geq \frac{\lambda_{2} k}{C-\lambda_{2}}$, when $0<\Delta k<\frac{\lambda_{2} k}{C-\lambda_{2}}$ and $\Delta k \geq \frac{\lambda_{2} k}{C-\lambda_{2}}$, conclusion can be similar to solve:

$$
Q^{*}= \begin{cases}Q_{3}^{*}=\bar{Q}, & 0<\Delta k<\frac{\lambda_{2} k}{C-\lambda_{2}}(\text { shown in figure 4) } \\ Q_{4}^{*}=\bar{Q}-\eta_{2}, & \Delta k \geq \frac{\lambda_{2} k}{C-\lambda_{2}}(\text { shown in figure 5) }\end{cases}
$$

The optimal retail price is:

$$
p^{*}=\left\{\begin{array}{lrl}
p_{3}^{*}=\bar{p}-\frac{\Delta k \bar{p}}{k+\Delta k}, & 0<\Delta k<\frac{\lambda_{2} k}{C-\lambda_{2}} \\
p_{4}^{*}=\bar{p}+\frac{\eta_{2}-\Delta k \bar{p}}{k+\Delta k}, & \Delta k \geq \frac{\lambda_{2} k}{C-\lambda_{2}}
\end{array}\right.
$$

Optimal profit is:

$$
\prod\left(Q^{*}\right)=\left\{\begin{array}{cr}
\prod\left(Q_{3}^{*}\right)=\overline{\prod_{\text {max }}(q)}-\frac{\Delta k \bar{P} \bar{Q}}{k+\Delta k}, & 0<\Delta k<\frac{\lambda_{2} k}{C-\lambda_{2}} \\
\prod\left(Q_{4}^{*}\right)=\overline{\prod_{\text {max }}(q)}-\eta_{2}(\bar{P}-C)+\frac{\eta_{2}-\Delta k \bar{p}}{k+\Delta k}\left(\bar{Q}-\eta_{2}\right) & \\
-\lambda_{2} \eta_{2}-\lambda \eta_{2}^{2}, & \Delta k \geq \frac{\lambda_{2} k}{C-\lambda_{2}}
\end{array}\right.
$$

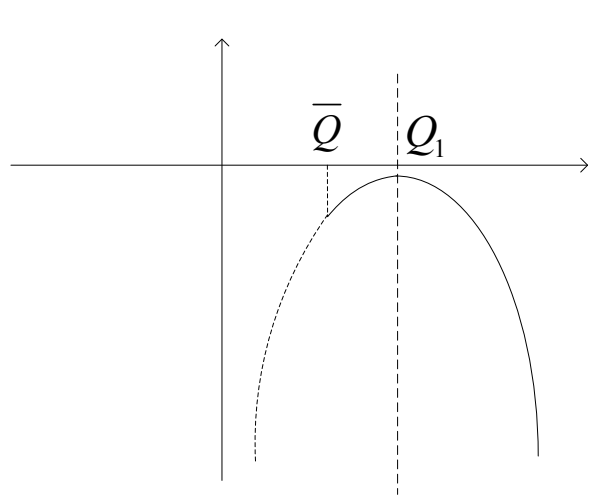

Figure 2.

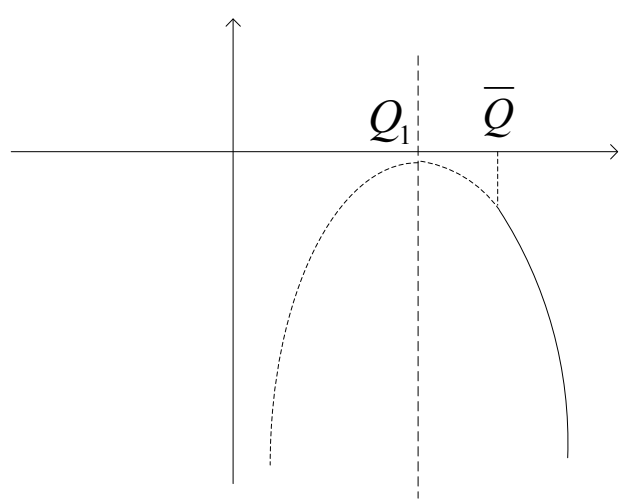

Figure 3.

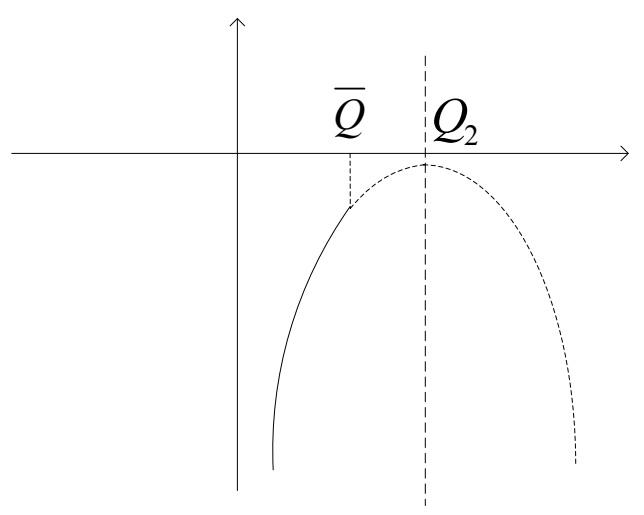

Figure 4.

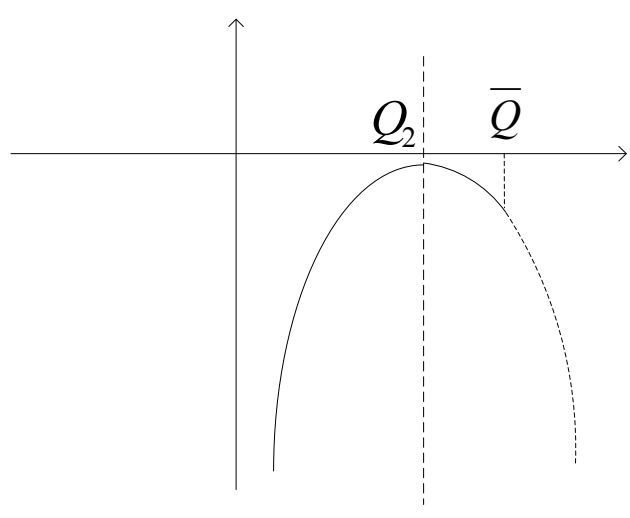

Figure 5. 
Summarize the above results:

Theorem 3. The sensitivity coefficient of the price-demand function (k) is changed $\Delta k$ by Emergencies, And Price-demand function is: $d=D-(k+\Delta k) p$. While retailer's retail price is $p^{*}$, the order quantity is $Q^{*}$, the profit of the entire supply chain is maximized, In other words, the centralized supply chain is optimal responded to emergencies.

$$
p^{*}=\left\{\begin{array}{l}
\bar{p}+\frac{\eta_{1}-\Delta k \bar{p}}{k+\Delta k},-k<\Delta k \leq \frac{-\lambda_{1} k}{C+\lambda_{1}} \\
\bar{p}-\frac{\Delta k \bar{p}}{k+\Delta k}, \frac{-\lambda_{1} k}{C+\lambda_{1}}<\Delta k<\frac{\lambda_{2} k}{C-\lambda_{2}} \\
\bar{p}+\frac{\eta_{2}-\Delta k \bar{p}}{k+\Delta k}, \Delta k \geq \frac{\lambda_{2} k}{C-\lambda_{2}}
\end{array}, Q^{*}=\left\{\begin{array}{l}
\bar{Q}-\eta_{1},-k<\Delta k \leq-\frac{\lambda_{1} k}{C+\lambda_{1}} \\
\bar{Q} \quad,-\frac{\lambda_{1} k}{C+\lambda_{1}}<\Delta k \frac{\lambda_{2} k}{C-\lambda_{2}} \\
\bar{Q}-\eta_{2}, \Delta k \geq \frac{\lambda_{2} k}{C-\lambda_{2}}
\end{array}\right.\right.
$$

Here $\eta_{1}=\frac{\left(C+\lambda_{1}\right) \Delta k+\lambda_{1} k}{2 \lambda(k+\Delta k)+2}, \eta_{2}=\frac{\left(C-\lambda_{2}\right) \Delta k-\lambda_{2} k}{2 \lambda(k+\Delta k)+2}$

Assumption: If $C-\lambda_{2}>\lambda(D-k C)$, suppose $\Delta k<\frac{(D-k C)(\lambda k+1)+\lambda_{2} k}{\left(C-\lambda_{2}\right)-\lambda(D-k C)}$.

Here, a necessary condition of the entire supply chain to maximize profit is: the optimal order quantity $Q^{*}>0$,

which can be expressed as $Q_{4}^{*}=\bar{Q}-\eta_{2}>0$. From the assumption, we can see that the condition is true.

We found that the optimal strategy responding to the change of sensitive coefficient of the price demand $(\Delta k)$ is: The retailer's retail price must be made a positive adjustment, the order quantity must be adjusted only while $\Delta k$ is beyond a certain range, If central decision-maker does not make a positive adjustment for retail price when unexpected events occur. The actual demand is $d=D-(k+\Delta k) \bar{p}$, Thus the retailer's order quantity is $\hat{Q}=d=\bar{Q}-\Delta k \bar{p}$, if $D-(k+\Delta k) \bar{p}>0$, which can be expressed as $\Delta k<\bar{Q} / \bar{p}$, the supply chain profit is:

$$
\prod(\hat{Q})=\hat{Q}(\bar{P}-C)-\lambda_{1}(\Delta k \bar{p})^{-}-\lambda_{2}(\Delta k \bar{p})^{+}-\lambda(\Delta k \bar{p})^{2}
$$

If $D-(k+\Delta k) \bar{p} \leq 0$, which can be expressed as $\Delta k \geq \bar{Q} / \bar{p}$, the order quantity is $\hat{Q}=0$, retailer Quit from the supply chain, Supplier's profit is : $-\lambda_{2} \bar{Q}-\lambda(\bar{Q})^{2}$. We consider (9) in two cases ( $\left.\Delta k<0, \Delta k \geq 0\right)$. So we have:

$$
\prod(\hat{Q})=\left\{\begin{array}{lc}
\overline{\prod_{\text {max }}(q)}-\Delta k \bar{P}(\bar{P}-C)+\lambda_{1} \Delta k \bar{p}-\lambda(\Delta k \bar{p})^{2}, & -k<\Delta k<0 \\
\overline{\prod_{\text {max }}(q)}-\Delta k \bar{P}(\bar{P}-C)-\lambda_{2} \Delta k \bar{p}-\lambda(\Delta k \bar{p})^{2}, & 0 \leq \Delta k<\frac{\bar{Q}}{\bar{p}} \\
-\lambda_{2} \bar{Q}-\lambda(\bar{Q})^{2}, & \Delta k \geq \frac{\bar{Q}}{\bar{p}}
\end{array}\right.
$$

\subsection{Numerical Examples}

We know that the profit generated by the optimal response to emergencies $\Pi(Q)$ is greater than $\prod(\hat{Q})$,

Let $k=4, C=8, D=300, \lambda_{1}=3, \lambda_{2}=2, \lambda=0.5$, computing by Matlab and drawing the figure, 
shown in Figure 6. We can see while $\Delta k$ is changed within the meaningful region, the change of the supply chain profit $\Pi(Q)$ and the profit $\Pi(\hat{Q})$ with the retail price unchanged.

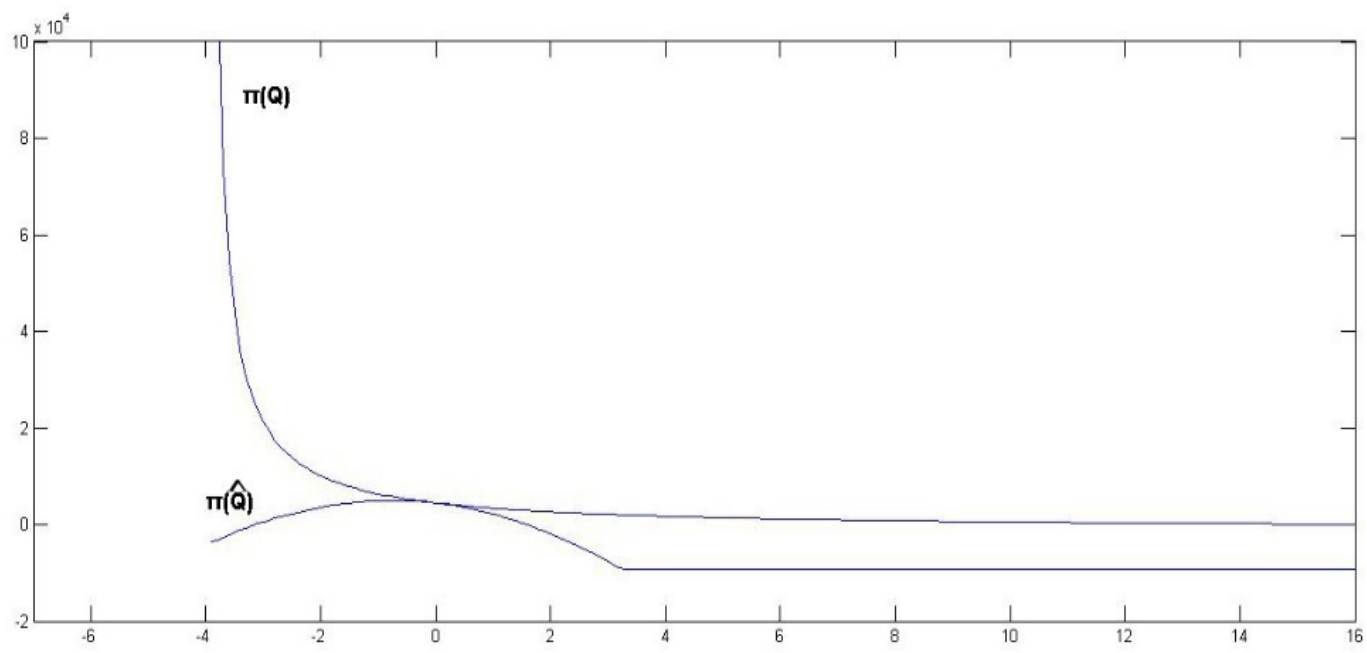

Figure 6. The change of $\Pi(Q)$ and $\Pi(\hat{Q})$

While $\Delta k$ is changed within the meaningful region, from the change of the supply chain profit $\Pi(Q)$ and the profit $\prod(\hat{Q})$ with the retail price unchanged in Figure 6, we have:

1) while $\Delta k$ is changed within the meaningful region after the emergencies, the optimal supply chain profit $\Pi(Q)$ with adjustment is always better than the profit $\prod(\hat{Q})$ with the retail price unchanged. which can be expressed as: while $\Delta k$ changed by emergencies, it is necessary to take appropriate coordination mechanism,

2) when $-k<\Delta k<-\lambda_{1} k /\left(C+\lambda_{1}\right)$, the optimal supply chain profit $\prod(Q)$ with adjustment is obviously much better than the profit $\prod(\hat{Q})$ with the retail price unchanged, At this time people's demand for supplies is no longer or less influenced by the price factor, which can be expressed as: while $k-\Delta k$ is significantly reduced, it is necessary to make a corresponding adjustment. For example, the demand for drugs during "5.12" earthquake period, the sensitivity coefficient of demand is less influenced by the price factor, improve the retail price and production is the optimal response to the pharmaceutical supply chain (If only from the supply chain's point of view);

3) when $-\lambda_{1} k /\left(C+\lambda_{1}\right)<\Delta k<\bar{Q} / \bar{p}$, the optimal supply chain profit $\prod(Q)$ with adjustment is better than the profit $\prod(\hat{Q})$ with the retail price unchanged, and equal to each other near the $\Delta k=0$, which can be expressed as: while sensitivity coefficient of the price-demand function $k$ unchanged or little changed, a useful strategy to the single-cycle supply chain is timely adjust the retail price ; 
4) when $\Delta k \geq \bar{Q} / \bar{p}$, the optimal supply chain profit $\Pi(Q)$ with adjustment is higher than the profit $\prod(\hat{Q})$ with the retail price unchanged, However, it is not much higher, and the two curves are almost parallel,

While $\Delta k$ increased to a certain value, the optimal order quantity will be reduced with the increase of $\Delta k$, which make the optimal supply chain profit $\Pi(Q)$ with adjustment decreases and tends to zero, and the profit $\prod(\hat{Q})$ with the retail price unchanged is always $\lambda_{2} \bar{Q}+\lambda(\bar{Q})^{2}$, which can be expressed as : while the retail price is constant, there is not any order which caused the loss of supplier,

\section{Supply Chain Coordination Mechanism to the Decentralized Decision-making with Emergencies}

Theorem 1 has indicated that the quantity discount contract and sharing Contract can encourage retailer to order $Q$ in general, and then achieved the coordination of the three-level supply chain composed by the supplier-government-Retailer, However, members of the supply chain often have different interests, Supplier, government and retailer make decisions in a decentralized way under the disrupted market scale and production cost, the previously derived coordination scheme must be modified to achieve the maximum supply chain profit, Recall that the optimal order quantity $Q^{*}$ and retail price $p^{*}$ are given in Theorem 1 for this case,

The following will discuss how to design a new program as quantity discounts and revenue sharing contract to coordinate the supply chain with the demand change caused by the change of the sensitivity coefficient of the price-demand function under unexpected emergencies, Accordingly, there are four situations,

4.1 Case 1: $-k<\Delta k \leq-\lambda_{1} k /\left(C+\lambda_{1}\right)$

When $-k<\Delta k \leq-\lambda_{1} k /\left(C+\lambda_{1}\right)$, in order to maximize supply chain profit, From Theorem 3 we know that the retailer's optimal order quantity is: $Q_{i}^{*}=\bar{Q}-\eta_{1}=(D-k C) / 2-\left[\left(C+\lambda_{1}\right) \Delta k+\lambda_{1} k\right] /[2 \lambda(k+\Delta k)+2]$. The profit of supply chain is: $\prod\left(Q_{1}^{*}\right)=\overline{\prod_{\max }(q)}+\left(\bar{Q}-\eta_{1}\right)\left(\eta_{1}-\Delta k \bar{P}\right) /(k+\Delta k)-\eta_{1}(\bar{P}-C)+\lambda_{1} \eta_{1}-\lambda \eta_{1}{ }^{2}$. suppose that the supplier's expect profit is $\prod_{s}\left(Q_{1}^{*}\right)=\left(1-\Phi_{1}\right)\left(1-\Phi_{2}\right) \prod\left(Q_{1}^{*}\right)$, and the government's expect profit is $\prod_{g}\left(Q_{1}^{*}\right)=\Phi_{1}\left(1-\Phi_{2}\right) \prod\left(Q_{1}^{*}\right)$, here: $\Phi_{1}, \Phi_{2} \in[0,1]$, So the following is Theorem 4 which give a supply chain coordination mechanism in the case of $-k<\Delta k \leq-\lambda_{1} k /\left(C+\lambda_{1}\right)$.

Theorem 4: when $-k<\Delta k \leq-\lambda_{1} k /\left(C+\lambda_{1}\right)$, the supplier's expect profit is $\prod_{s}\left(Q_{1}^{*}\right)=\left(1-\Phi_{1}\right)\left(1-\Phi_{2}\right) \prod\left(Q_{1}^{*}\right) \quad$ and $\quad$ the government's expect profit is $\prod_{g}\left(Q_{1}^{*}\right)=\Phi_{1}\left(1-\Phi_{2}\right) \prod\left(Q_{1}^{*}\right) \quad, \quad$ the quantity discounts and revenue sharing contract $\left(W_{1}\left(Q_{1}^{*}\right), \Phi_{1} ; W_{2}\left(Q_{1}^{*}\right), \Phi_{2}\right)$ can be used to coordinate the supply chain.

Where

$$
W_{1}\left(Q_{1}^{*}\right)=\Phi_{1}\left(C_{s}+C_{g}\right)-C_{g}-\Phi_{1}\left(\lambda_{1} \eta_{1}-\lambda \eta_{1}^{2}\right) /\left(\bar{Q}-\eta_{1}\right)
$$


$W_{2}\left(Q_{1}^{*}\right)=\Phi_{2} C-C_{r}-\frac{\Phi_{2}\left(\lambda_{1} \eta_{1}-\lambda \eta_{1}^{2}\right)}{\bar{Q}-\eta_{1}} ;$ at the same time, $W_{1}\left(Q_{1}^{*}\right), W_{2}\left(Q_{1}^{*}\right)$ are positive numbers, $\Phi_{1}, \Phi_{2} \quad$ should meet with:

$\Phi_{2}>\frac{\left(\bar{Q}-\eta_{1}\right) C_{r}}{\left(\bar{Q}-\eta_{1}\right) C-\lambda_{1} \eta_{1}+\lambda \eta_{1}^{2}}$,

Proof that omitted,

4.2 Case 2: $-\lambda_{1} k /\left(C+\lambda_{1}\right)<\Delta k \leq 0$

When $-\lambda_{1} k /\left(C+\lambda_{1}\right)<\Delta k \leq 0$, similarly, the optimal order quantity is: $Q_{i}^{*}=\bar{Q}=(D-k C) / 2$. The

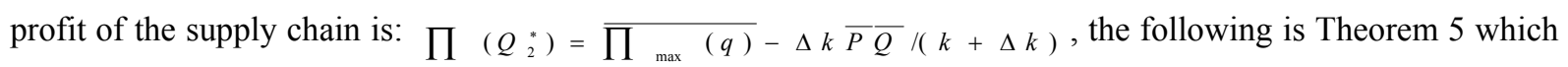
give a supply chain coordination mechanism in the case of $-\lambda_{1} k /\left(C+\lambda_{1}\right)<\Delta k \leq 0$,

Theorem 5: when $-\lambda_{1} k /\left(C+\lambda_{1}\right)<\Delta k \leq 0$, the supplier's expect profit is $\prod_{s}\left(Q_{2}^{*}\right)=\left(1-\Phi_{1}\right)\left(1-\Phi_{2}\right) \prod\left(Q_{2}^{*}\right) \quad$,and the government's expect profit is $\prod_{g}\left(Q_{2}^{*}\right)=\Phi_{1}\left(1-\Phi_{2}\right) \prod\left(Q_{2}^{*}\right) \quad, \quad$ the quantity discounts and revenue sharing contract $\left(W_{1}\left(Q_{2}^{*}\right), \Phi_{1} ; W_{2}\left(Q_{2}^{*}\right), \Phi_{2}\right) \quad$ can be used to coordinate the supply chain. Where $W_{1}\left(Q_{2}^{*}\right)=\Phi_{1}\left(C_{s}+C_{g}\right)-C_{g}, W_{2}\left(Q_{2}^{*}\right)=\Phi_{2} C-C_{r}$; at the same time, $W_{1}\left(Q_{2}^{*}\right), W_{2}\left(Q_{2}^{*}\right)$ are positive numbers, $\Phi_{1}, \Phi_{2}$ should meet with: $\Phi_{1}>C_{g} /\left(C_{s}+C_{g}\right), \Phi_{2}>C_{r} / C$.

Proof that omitted,

4.3 Case 3: $0<\Delta k<\lambda_{2} k /\left(C-\lambda_{2}\right)$

When $0<\Delta k<\lambda_{2} k /\left(C-\lambda_{2}\right)$, similarly, the optimal order quantity is: $Q_{3}^{*}=\bar{Q}=(D-k C) / 2$. The profit of the supply chain is: $\prod\left(Q_{3}^{*}\right)=\overline{\prod_{\text {max }}(q)}-\Delta k \bar{P} \bar{Q} /(k+\Delta k)$, the following is Theorem 6 which gives a supply chain coordination mechanism in the case of $0<\Delta k<\lambda_{2} k /\left(C-\lambda_{2}\right)$.

Theorem 6: when $0<\Delta k<\lambda_{2} k /\left(C-\lambda_{2}\right)$, the supplier's expect profit is $\prod_{s}\left(Q_{3}^{*}\right)=\left(1-\Phi_{1}\right)\left(1-\Phi_{2}\right) \prod\left(Q_{3}^{*}\right) \quad$ and $\quad$ the government's expect profit is $\prod_{g}\left(Q_{3}^{*}\right)=\Phi_{1}\left(1-\Phi_{2}\right) \prod\left(Q_{3}^{*}\right) \quad, \quad$ the quantity discounts and revenue sharing contract $\left(W_{1}\left(Q_{3}^{*}\right), \Phi_{1} ; W_{2}\left(Q_{3}^{*}\right), \Phi_{2}\right) \quad$ can be used to coordinate the supply chain. Where $W_{1}\left(Q_{3}^{*}\right)=\Phi_{1}\left(C_{s}+C_{g}\right)-C_{g}, W_{2}\left(Q_{3}^{*}\right)=\Phi_{2} C-C_{r} ;$ at the same time, $W_{1}\left(Q_{2}^{*}\right), W_{2}\left(Q_{2}^{*}\right)$ are positive 
numbers, $\Phi_{1}, \Phi_{2}$ should meet with: $\Phi_{1}>C_{g} /\left(C_{s}+C_{g}\right), \Phi_{2}>C_{r} / C$,

Proof that omitted,

4.4 Case 4: $\Delta k \geq \lambda_{2} k /\left(C-\lambda_{2}\right)$

When $\Delta k \geq \lambda_{2} k /\left(C-\lambda_{2}\right)$, similarly, the optimal order quantity is: $Q_{4}^{*}=\bar{Q}-\eta_{2}$, The profit of the supply chain is $\prod\left(Q_{4}^{*}\right)=\overline{\prod_{\text {max }}(q)}+\left(\bar{Q}-\eta_{2}\right)\left(\eta_{2}-\Delta k \bar{P}\right) /(k+\Delta k)-\eta_{2}(\bar{P}-C)-\lambda_{2} \eta_{2}-\lambda \eta_{2}^{2}$, the following is Theorem 7 which give a supply chain coordination mechanism in the case of $\Delta k \geq \lambda_{2} k /\left(C-\lambda_{2}\right)$.

Theorem 7: when $\Delta k \geq \lambda_{2} k /\left(C-\lambda_{2}\right) \quad, \quad$ the supplier's expect profit is
$\prod_{s}\left(Q_{4}^{*}\right)=\left(1-\Phi_{1}\right)\left(1-\Phi_{2}\right) \prod\left(Q_{4}^{*}\right) \quad, \quad$ and the government's expect profit is $\prod_{g}\left(Q_{4}^{*}\right)=\Phi_{1}\left(1-\Phi_{2}\right) \prod\left(Q_{4}^{*}\right) \quad, \quad$ the quantity discounts and revenue sharing contract $\left(W_{1}\left(Q_{4}^{*}\right), \Phi_{1} ; W_{2}\left(Q_{4}^{*}\right), \Phi_{2}\right) \quad$ can $\quad$ be $\quad$ used to coordinate the supply chain. Where $W_{1}\left(Q_{4}^{*}\right)=\Phi_{1}\left(C_{s}+C_{g}\right)-C_{g}+\Phi_{1}\left(\lambda_{2} \eta_{2}+\lambda \eta_{2}^{2}\right) /\left(\bar{Q}-\eta_{2}\right)$ $W_{2}\left(Q_{4}^{*}\right)=\Phi_{2} C-C_{r}+\Phi_{2}\left(\lambda_{2} \eta_{2}+\lambda \eta_{2}^{2}\right) /\left(\bar{Q}-\eta_{2}\right) ;$ at the same time, are positive numbers, $\Phi_{1}, \Phi_{2}$ should meet with :

$$
\Phi_{1}>\frac{\left(\bar{Q}-\eta_{2}\right) C_{g}}{\left(\bar{Q}-\eta_{2}\right)\left(C_{s}+C_{g}\right)+\lambda_{2} \eta_{2}+\lambda \eta_{2}^{2}}, \Phi_{2}>\frac{\left(\bar{Q}-\eta_{2}\right) C_{r}}{\left(\bar{Q}-\eta_{2}\right) C+\lambda_{2} \eta_{2}+\lambda \eta_{2}^{2}},
$$

Proof that omitted.

Definition 2: If a contract can coordinate the supply chain around the emergencies. Then this contract equips with the anti-disruption-ability.

From Theorem 1 to Theorem 7, we have the following proposition.

Proposition 1: the quantity discounts and revenue sharing contract $\left(W_{1}, \Phi_{1} ; W_{2}, \Phi_{2}\right)$ equips with the anti-disruption-ability.

\section{Conclusion and Discussion}

This paper studies the government and enterprise joint reserve sales emergency supplies mode, which can be expressed as that the government (or through commission, holding a third party logistics company) orders emergency supplies from the enterprise and sells dynamically in the effective period of the quality of the materials. This one is different from the government's past single concentration reserve mode, it reduces the government budget, avoids the severe supply losses of the expired materials which are discarded as useless, can realize the win-win situation between government, the suppliers and the retailers.

On one hand, using Stackelberg game theory analysis, we can find that if the government would take the past single concentration reserve mode, it is not only impossible for it to have economic income, increase the fiscal budget, but also could cause a lot of stock wasted after expired and influence the economic development. While the government and enterprises cooperate and involve in the three-level suppliers-the government-retailers supply chain, it can not only reduce the financial budget, avoid the waste of inventory expires, but can increase the fiscal revenue and realize the coordination of the supply chain.

On the other hand, based on the three-level supply chain joint by the government and enterprises, we find an optimal strategy for emergency of a single coordinating cycle supply chain. For centralized supply chain, we compare the profits of the optimal strategy and the unchanged one through calculating, and give its optimal strategy for emergencies, For the decentralized supply chain system, according to the different degrees of disturbance, we put forward the optimal emergency coordination mechanism. Research shows that, after the adjustment the quantity discount and revenue sharing contract we can not only coordinate emergencies, but 
coordinate the supply chain whose $\Delta k=0$.

\section{References}

Cachon G. P. (2003). Supply Chain Coordination with Contracts. Handbooks in OR \& MS, 11, 229-339.

Chen, H. T., \& Li, C. Y. (2007). Coordination and revenue sharing contract with newsvendor problem. ICM CE 2007. New York: IEEE, (5), 585-590.

Clark A. J., \& Scarf H. (1960). Optimal Policies for A MultiEchelon Inventory Problem. Management Science, (6), 475-490. http://dx.doi.org/10.1287/mnsc.6.4.475

Jeuland A. L., \& Shugan S. M. (1983). Managing channel profits. Marketing Science, 2(3), 239-272. http://dx.doi.org/10.1287/mksc.2.3.239

Lei, Dong, Gao, Chengxiu, \& Li, Jianbin. (2006). Supply Chain Coordination with Demand and Production Cost Disruptions. Systems Engineering-Theory \& Practice, 26(9), 51-59.

Pasternack B. A. (1985). Optimal Pricing and Returns Policies for Perishable Commodities. Marketing Science, 4, 166-176. http://dx.doi.org/10.1287/mksc.4.2.166

Qi X. T., Jonathan F. B., \& Yu G. (2004). Supply chain coordination with demand disruptions. Omega, 32(4), 301-312. http://dx.doi.org/10.1016/j.omega.2003.12.002

Wang, R., Ji, J. H., \& Ying, S. H. (2006). Analysis of revenue sharing contract with uncertain demand in supply chain. ICM CE 2006. New York: IEEE, 522-525.

Xin, C. L., Ma, W. M., \& Liu, B. (2007). Competitive revenue sharing contract. Machine L earning \& Cyber netics, 3(11), 1687-1692.

Yang, Deli, Guo, Qiong, He, Yong, \& Xu, Jingyi. (2006). Review of Supply Chain Contracts. Chinese Journal of Management, 3(1), 117-125.

Yang, Z. H., Chen, H., Zhao, Q, et al. (2007). Supply chain coordination under demand and production cost disruptions with revenue sharing contract. Service Systems and Service Management. New York: IEEE, 1-5.

Yang, Zhihui, Chen, Hong, Zhao Qian, \& Ma, Ronghua. (2010). Study on Supply Chain Ripple Effect under Stochastic Demand and Production Cost Disruptions. Chinese Journal of Management, 7(5), 728-732.

Zhang, Handong, Wang, Qingyun, \& Cen, Yuwan. (2009). EOQ model for deteriorating items under elastic demand. Computer Integrated Manufacturing Systems, 15(8), 1528-1533. 